\title{
Validation of the Publication of New Names and New Combinations Previously Effectively Published Outside the IJSB
}

\author{
List No. $43 \dagger$
}

The purpose of this announcement is to effect the valid publication of the following new names and new combinations under the procedure described previously [Int. J. Syst. Bacteriol. 27(3):iv, 1977]. Authors and other individuals wishing to have new names and/or combinations included in future lists should send the pertinent reprint or a photocopy thereof to the IJSB (c/o ASM) for confirmation that all of the other requirements for valid publication have been met. It should be noted that the date of valid publication of these new names and combinations is the date of publication of this list, not the date of the original publication of the names and combinations. The authors of the new names and combinations are as given below, and these authors' names will be included in the author index of the present issue and in the volume author index in this issue of the IJSB.

Inclusion of a name on these lists validates the name and thereby makes it available in bacteriological nomenclature. The inclusion of a name on this list is not to be construed as taxonomic acceptance of the taxon to which the name is applied. Indeed, some of these names may, in time, be shown to be synonyms, or the organisms may be transferred to another genus, thus necessitating the creation of a new combination.

\begin{tabular}{|c|c|c|c|c|}
\hline Name & Proposed as: & Author(s) (reference) & Priority $^{a}$ & Nomenclatural type ${ }^{b}$ \\
\hline Acetobacter europaeus & New species & Sievers et al. (2) & 2 & Strain DES 11 (= DSM 6160) \\
\hline Aquifex & New genus & Huber et al. (1) & 2 & Aquifex pyrophilus \\
\hline Aquifex pyrophilus & New species & Huber et al. (1) & 2 & Strain Kol5a (= DSM 6858) \\
\hline $\begin{array}{l}\text { Bifidobacterium pseudolongum } \\
\text { subsp. globosum (basonym: } \\
\text { Bifidobacterium globosum) }\end{array}$ & New combination & Yaeshima et al. (4) & 2 & Strain RU 224 (= ATCC 25865) \\
\hline $\begin{array}{l}\text { Bifidobacterium pseudolongum } \\
\text { subsp. pseudolongum }\end{array}$ & New subspecies & Yaeshima et al. (4) & 2 & Strain PNC-2-9G (= ATCC 25526) \\
\hline Micromonospora chersina & New species & Tomita et al. (3) & 1 & Strain M956-1 (= ATCC 53710) \\
\hline
\end{tabular}

† Lists 1 through 42 were published in the Int. J. Syst. Bacteriol. 27:306, 1977; 29:79, 436, 1979; 30:601, 676, 1980; 31:215, 382, 1981; 32:266, 384, 1982; 33:438, $672,896,1983 ; \mathbf{3 4 : 9 1}, 270,355,503,1984 ; 35: 223,375,535,1985 ; 36: 354,489,573,1986 ; 37: 179,1987 ; 38: 136,220,328,449,1988 ; 39: 93,205,371,495,1989 ; 40: 105$, 212, 320, 470, 1990; 41:178, 331, 456, 580, 1991; and 42:191, 327, 511, 1992.

${ }^{a}$ Priority number assigned according to the date the documentation and request for validation are received.

${ }^{b}$ Abbreviations: ATCC, American Type Culture Collection, Rockville, Md.; DSM, Deutsche Sammlung von Mikroorganismen und Zellkulturen GmbH, Braunschweig, Germany.

Notification that New Names and New Combinations Have Appeared in the IJSB ${ }^{a}$

\begin{tabular}{|c|c|c|c|}
\hline Name & Proposed as: & Author(s) & $\begin{array}{l}\text { IJSB reference } \\
\text { description }\end{array}$ \\
\hline $\begin{array}{l}\text { Arcobacter } \\
\text { Arcobacter skirrowii } \\
\text { Arcobacter butzleri (basonym: } \\
\quad \text { Campylobacter butzleri) }\end{array}$ & $\begin{array}{l}\text { emend. } \\
\text { sp. nov. } \\
\text { comb. nov. }\end{array}$ & $\begin{array}{l}\text { Vandamme et al. } \\
\text { Vandamme et al. } \\
\text { Vandamme et al. }\end{array}$ & $\begin{array}{l}42(3): 355 \\
42(3): 355 \\
42(3): 355\end{array}$ \\
\hline Mycoplasma penetrans & sp. nov. & Lo et al. & $42(3): 363$ \\
\hline Enterococcus flavescens & sp. nov. & Pompei et al. & $42(3): 367$ \\
\hline Borrelia burgdorferi & emend. & Baranton et al. & $42(3): 382$ \\
\hline Borrelia garinii & sp. nov. & Baranton et al. & $42(3): 382$ \\
\hline Anaerovibrio burkinabensis & sp. nov. & Ouattara et al. & $42(3): 395$ \\
\hline Desulfovibrio longus & sp. nov. & Magot et al. & 42(3):402 \\
\hline Legionella shakespearei & sp. nov. & Verma et al. & $42(3): 406$ \\
\hline Porphyromonas circumdentaria & sp. nov. & Love et al. & $42(3): 435$ \\
\hline $\begin{array}{l}\text { Porphyromonas salivosa (basonym: } \\
\text { Bacteroides salivosus) }\end{array}$ & comb. nov. & Love et al. & $42(3): 438$ \\
\hline Bacillus methanolicus & sp. nov. & Arfman et al. & $42(3): 444$ \\
\hline Flexibacter ovolyticus & sp. nov. & Hansen et al. & $42(3): 457$ \\
\hline $\begin{array}{l}\text { Methanothrix (pro synon. } \\
\text { Methanosaeta) thermophila }\end{array}$ & sp. nov. & Komagata et al. & $42(3): 465$ \\
\hline Lactobacillus gallinarum & sp. nov. & Fujisawa et al. & $42(3): 489$ \\
\hline Lactobacillus johnsonii & sp. nov. & Fujisawa et al. & 42(3):489 \\
\hline
\end{tabular}

a This listing of names published in a previous issue of IJSB is provided as a service to bacteriology to assist in the recognition of new names and new descriptions. This procedure was proposed by the Judicial Commission [Minute 11 (ii), Int. J. Syst. Bacteriol. 41:185, 1991]. The names given herein have priority according to the issue of the IJSB in which they were published. 